\title{
Why New Journals? The Growth of the EJNMMI Family
}

\author{
Ignasi Carrió $^{1} \cdot$ Arturo Chiti $^{2}$
}

Published online: 12 August 2015

(C) Springer-Verlag Berlin Heidelberg 2015

As only published data exist, publishing has become a crucial step in scientific research. However, publishing information is more than just making it publicly available. Researchers must prepare their manuscripts in such a way as to clearly demonstrate the value of the research, they have to discuss the implications for science and clinical medicine, and they must draw appropriate conclusions. Upon submission, peerreviewed journals will evaluate the quality and strength of the data, the originality and clinical relevance of the research, and may publish the manuscripts that meet all the standards of such journals. After publication, the importance of the published material will be demonstrated by the numbers of citations and downloads, by the reproducibility of the results by others, and by the impact on clinical practice or research.

Over the years the number of scientific journals has increased exponentially, with now more than 40000 journals worldwide offering opportunities to scientific researchers for publication of their work. Currently, more than 500000 papers a year enter PubMed, a number that is expected to rise as emerging countries increase their contribution to scientific production. Furthermore, the internet has revolutionized the way we access information in science and medicine. Access to massive amounts of scientific data is universal. Efficient search engines in Google or PubMed allow us to find what

Ignasi Carrió

icarrio@santpau.cat

Arturo Chiti

arturo.chiti@cancercenter.humanitas.it

1 Department of Nuclear Medicine, Autonomous University of Barcelona, Hospital Sant Pau, Sant Quintí 89, 08025 Barcelona, Spain

2 Nuclear Medicine, IRCCS Humanitas, Milan, Italy we are seeking in a few clicks. Often, downloading review papers or guidelines may suffice to give us a good perspective on a given problem, circumventing the need to carefully study large numbers of single-center papers or original scientific reports. Despite the many tools to help us cope with the excess of available scientific materials, how best to extract the relevant information from the thousands of journals remains a challenge for scientists and physicians.

Given these impressive numbers, do we really need yet more journals? Or, in different words, why is the European Journal of Nuclear Medicine and Molecular Imaging (EJNMMI) developing a family of journals in nuclear medicine and molecular imaging? One could easily argue that we already have enough opportunities for publication and therefore there is no need for further journals.

However, science in medicine is growing in such a way that specialties and subspecialties are developing even within a well-established field such as nuclear medicine and molecular imaging. In many areas of science and medicine, there are an increasing number of fields which, despite belonging to the same scientific community, work on different topics and often have their own distinct language. In nuclear medicine and molecular imaging, basic scientists, physicists, radiopharmacists, and others have grown into communities in their own right, with specific educational and scientific needs. Although some of their research articles are of general interest for physicians and scientists working in nuclear medicine, others are read only by certain specialists.

Objective numbers indicate that the EJNMMI, the flagship journal of nuclear medicine and molecular imaging in Europe, gives only limited opportunities for publication to all these communities within our specialty. In fact, the EJNMMI has been receiving ever-increasing numbers of submitted manuscripts, while the acceptance rate has declined significantly. 
Over 1200 original scientific articles were submitted to the EJNMMI last year, with a resulting rejection rate close to $80 \%$. Often, good papers that genuinely deserve to be published are declined because of space restrictions or because "limited" priority has been assigned after peer review. Obviously the assigned priority is influenced by the interest of papers for a broad readership, which puts highly specialized or technical papers at a disadvantage compared with manuscripts that address issues of common interest to physicians or scientists in the field of nuclear medicine and molecular imaging. Therefore, good papers are sometimes declined not on grounds of quality, but rather because of their scope and contents. Such papers have value for physicians and scientists working in the field of nuclear medicine and deserve to be published.

In response to the increasing scientific and publishing needs of the communities within nuclear medicine and molecular imaging, the solution adopted by the EJNMMI, with the support of the EANM, has been to develop the EJNMMI family of journals. In addition to the mother journal, the family currently has two established journals, EJNMMI Research (started in 2011) and EJNMMI Physics (started in 2013), and a new one is ready to be launched, EJNMMI Radiopharmacy and Chemistry. The new journals in the family are named with the initials of the mother journal in order to take advantage of the prestige and reputation of EJNMMI as a brand name, and are intended to offer additional opportunities for publication to the respective scientific communities. The new e-only journals are published with Open Access and have independent peer review systems under the guidance of respected Editors in the fields concerned (Angelika Bischof-Delaloye, Thomas Beyer, and Philip Elsinga, respectively) (Fig. 1). With this model demonstrating success, opportunities may rise to add further journals to the family.

Authors may submit their manuscripts directly to any of the journals in the EJNMMI family. In addition, papers considered to be less suitable for publication in the main journal after peer review may be eligible for transfer to EJNMMI Research, EJNMMI Physics, or EJNMMI Radiopharmacy and Chemistry. In such cases the authors are offered the opportunity to resubmit to the companion journal, where they can find a fast-track option for reconsideration and potentially publication of their work.

In the meantime it is important to recall that the EJNMMI has an Impact Factor of 5.217. EJNMMI Research is now well established; it had $>300000$ downloads in 2014 and will have an Impact Factor in 2016. EJNMMI Physics had $>50000$ downloads in 2014 and will follow a similar evolution. It is important to recognize that in addition to the Impact Factor, downloads also reflect the importance and influence on clinical practice of scientific articles. We look forward to these new journals having significant Impact Factors in the near future, and we warmly welcome EJNMMI Radiopharmacy and Chemistry to the family and wish Philip Elsinga all possible success.
Fig. 1 The growing EJNMMI journal family

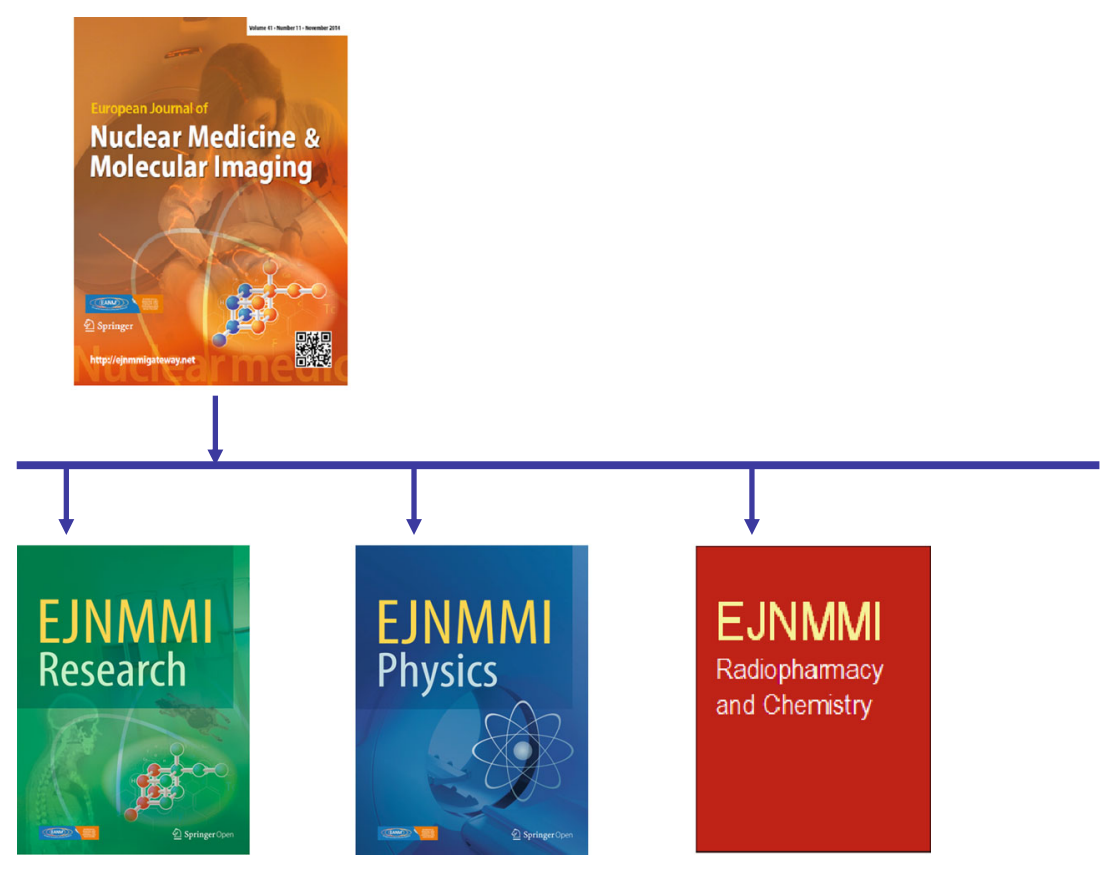

